\title{
EchoGéo
}

$17 \mid 2011$

Activités extractives

\section{Nouvelles géographies des activités extractives}

Introduction

\section{Géraud Magrin et Laetitia Perrier-Bruslé}

\section{(2) OpenEdition}

Journals

Édition électronique

URL : https://journals.openedition.org/echogeo/12586

DOI : 10.4000/echogeo.12586

ISSN : 1963-1197

Éditeur

Pôle de recherche pour l'organisation et la diffusion de l'information géographique (CNRS UMR 8586)

Référence électronique

Géraud Magrin et Laetitia Perrier-Bruslé, « Nouvelles géographies des activités extractives », EchoGéo

[En ligne], 17 | 2011, mis en ligne le 06 octobre 2011, consulté le 10 août 2021. URL : http://

journals.openedition.org/echogeo/12586 ; DOI : https://doi.org/10.4000/echogeo.12586

Ce document a été généré automatiquement le 10 août 2021.

EchoGéo est mis à disposition selon les termes de la licence Creative Commons Attribution - Pas d'Utilisation Commerciale - Pas de Modification 4.0 International (CC BY-NC-ND) 


\section{Nouvelles géographies des activités extractives}

Introduction

Géraud Magrin et Laetitia Perrier-Bruslé

\section{NOTE DE L'ÉDITEUR}

La coordination du dossier a été assurée par Géraud Magrin et Laetitia Perrier-Bruslé qui remercient l'ensemble des évaluateurs, ainsi que Nicolas Donner pour sa précieuse relecture de l'article introductif.

1 Après un premier dossier consacré aux mines d'Afrique de l'Ouest ${ }^{1}$, EchoGéo prolonge ici l'exploration de la géographie contemporaine des activités extractives en s'intéressant à de nouveaux espaces (Burkina Faso, Guyane, Gabon, Golfe de Guinée ; Asie du Sud et Afrique orientale) et à des ressources variées (or, fer, pétrole, gemmes). Les cinq textes qui composent le présent dossier invitent à l'exploration de lieux, de stratégies d'acteurs, de relations entre acteurs et entre acteurs et milieux. Dans tous ces processus, l'espace intervient comme dimension explicative ou contexte signifiant.

2 Ce texte liminaire se propose de mettre en perspective ces textes, en insistant sur les invariants et les nouveaux paramètres qui dessinent le contexte actuel de la géographie des activités extractives; puis, il présente et parfois discute l'apport spécifique de chacun de ces articles.

\section{Le socle extractif et les contradictions du monde}

Les activités extractives sentent le souffre ou le passé. À l'heure où l'apparente dématérialisation des économies, l'hypertrophie de la sphère numérique, l'ère de facebook et des économies de la connaissance valorisent l'immatériel, il n'est pas inutile de rappeler que, plus que jamais, tout commence, ou du moins tout est permis, par la mise en circulation de matières naturelles enfouies. La modernité liquide (Bauman, 
2006) a un besoin vital d'énergie et de matériaux solides. La mondialisation contemporaine est fille de l'alchimie qui a transformé les matières premières extraites des profondeurs du sous-sol en ressources (Raffestin, 1980). Celles-ci sont ainsi, au moins depuis la révolution industrielle du $\mathrm{XIX}^{\mathrm{e}}$ siècle, la base de fonctionnement $\mathrm{du}$ système monde (Gottmann, 1952). Elles fournissent à la fois l'essentiel de l'énergie (charbon, hydrocarbures, uranium) d'un monde qui en est de plus en plus boulimique, la matière des fabrications, lourdes (fer, cuivre, bauxite, etc.) ou légères (métaux et terres rares), ainsi que des supports d'usages symboliques d'importance variable (or, diamant, gemmes).

4 Mais l'époque est inquiète, sinon ingrate. Et ces activités extractives qui supportent les raffinements de la mondialisation contemporaine ont mauvaise presse. Elles sont associées aux dégradations multiformes de l'environnement: la consommation d'énergie carbonée est cause majeure du réchauffement climatique ; certaines matières inoffensives dans leur inertie deviennent dangereuses par leur extraction, leur transport ou leur usage ultérieur, qui rendent leurs " débordements » ${ }^{2}$ redoutables car difficilement contrôlables - au sens technique et géographique -, comme les pollutions marines par les hydrocarbures ou les accidents nucléaires; toutes menacent, dans des proportions variables, les environnements locaux dans lesquelles elles s'inscrivent, et ces atteintes sont de moins en moins tolérées. L'extraction des matières premières du sous-sol porte aussi un fardeau politique : c'est sur lui que repose l'idée de malédiction des ressources naturelles, qui associe en un solide cercle vicieux, pauvreté et richesse en ressources naturelles. Dans des pays aux institutions faibles, en particulier, les rentes extractives s'accompagneraient d'un ensemble de maux d'ordre macroéconomique (stagnation des secteurs productifs, endettement) et politique (autoritarisme, corruption, conflits) susceptibles d'entretenir le sous-développement (voir la synthèse critique de Rosser, 2006 ; Le Billon, 2005 sur les conflits, et Magrin, 2011). Enfin, la dissociation partielle entre pôles producteurs et consommateurs font des plus stratégiques de ces ressources un enjeu central des relations internationales et de leurs tensions (Gabriel-Oyhamburu, 2010).

5 La grande sensibilité des questions extractives reflète donc les contradictions sur lesquelles est construit le monde contemporain. Leur géographie et leur géopolitique associent des éléments stables, mais aussi des dynamiques nouvelles. Les textes de ce dossier, au-delà des situations spécifiques qu'ils décrivent, aident à en déchiffrer l'importance et la signification.

6 Ainsi, tout n'est pas nouveau dans la géographie des activités extractives. La géologie n'est pas sans inertie. Les grands producteurs des principales ressources extractives sont stables. Les géopolitiques qui y sont adossées ont leur permanence : les gisements géants d'hydrocarbures du Moyen Orient, du Maghreb, de Russie, du Venezuela, supportent des économies rentières et des systèmes politiques plus ou moins autoritaires, voire violents (Martinez, 2010). Ils sont au cœur de relations internationales caractérisées par une asymétrie dynamique entre les États, selon qu'ils disposent des ressources et / ou de la technologie pour les extraire, des deux ou d'aucun des deux, la maîtrise des capacités de mise en circulation constituant un atout central (Raffestin, 1980). Le texte de Romain Dittgen montre bien les modalités de ce face à face entre le géant chinois intéressé par le fer du petit Gabon. L'Afrique demeure jusqu'à présent dans son rôle historique de pourvoyeur dominé de ressources 
naturelles, notamment extractives, pour le système mondial, même si cette position n'exclut nullement une part - variable - d'autonomie (Bayart, 1989).

7 Le rapport aux ressources est en outre caractérisé par des phénomènes cycliques même si, ici comme ailleurs, l'histoire tient plus du solénoïde que de l'éternel recommencement. Ainsi de la peur de l'épuisement des ressources, qui s'exprime avec force lors des chocs pétroliers des années 1970, conduisant, longtemps avant la vogue de la décroissance, le Club de Rome à recommander une croissance zéro. Depuis la fin des années 1990, la croissance économique mondiale soutenue, tirée par les seuls pays émergents géants depuis la crise financière de 2008, stimule le cours des matières premières, notamment extractives. La fin des ressources aisément accessibles a des implications géographiques : elle déclenche la course à des ressources nouvelles jusque là protégées par l'hostilité de leurs environnements écologiques (pôles, offshore ultraprofond, forêts denses) ou géopolitiques. L'aventurisme extractif moderne, en abordant ces nouvelles frontières ${ }^{3}$, dans des espaces parfois sous surveillance de l'opinion publique mondiale pour leur haute valeur écologique, soulève donc de nouveaux risques, environnementaux et politiques, et donc de nouveaux dispositifs de gestion du risque et de protection des activités, comme le montre le texte de Nicolas Donner sur l'immunisation des enclaves pétrolières.

8 Cycliques aussi, les cours des matières premières entretiennent une relation dialectique avec les politiques étatiques de gestion des ressources. Chaque boom déclenche un regain de nationalisme extractif (énergétique ou minéral), aussi fragile que les rentes qui l'ont rendu possible. Les années 1960-70 voient la nationalisation des ressources dans certains grands pays producteurs d'hydrocarbures, la création de l'OPEP, l'affirmation internationale de certains pays grâce à leurs ressources extractives (comme le Gabon avec son pétrole ou le Zaïre de Mobutu grâce au cuivre). Les nationalismes pétroliers et miniers des années 2000 leur répondent, avec la nationalisation du gaz en Bolivie (Perrier-Bruslé, 2008), l'affirmation du Venezuela de Chavez, ou de la petite Guinée Équatoriale en Afrique centrale. Cependant, la relation est moins simple qu'elle n'y paraît. Le printemps arabe vient battre en brèche quelques idées reçues. Il rappelle que le pétrole, pas plus qu'aucune autre ressource, n'est l'horizon central de tous les soubresauts politiques et géopolitiques. La chute de Kadhafi serait-elle l'exception qui confirme la règle érigeant certains États pétroliers en «États faillis durables $»^{4}$ (Soares de Oliveira, 2007) ?

9 L'air du temps porte aussi des changements majeurs. La peur de la pénurie de ressources se conjugue avec celle des menaces globales, au premier rang desquelles figure le réchauffement climatique. La question de la viabilité tant locale que globale des relations entre sociétés et environnements a servi de matrice au paradigme du développement durable, désormais incontournable. Son influence sur la géographie mondiale des activités extractives reste à interpréter. En Amérique latine, et plus récemment en Afrique, les mouvements issus de la société civile, et parfois les riverains des sites extractifs (peuples autochtones et autres habitants concernés), relayés par des ONG nationales et internationales de défense des droits de l'Homme ou de l'environnement, s'appuient sur les préceptes du développement durable pour affirmer leurs droits de regard sur les activités des grandes compagnies extractives ou sur les politiques de cession des ressources naturelles des États. Des débats s'ouvrent au lieu du fait accompli de jadis pour limiter les impacts locaux négatifs de l'extraction et améliorer la transparence dans la gestion des rentes (voir Magrin, 2011). Les pressions 
conjuguées des riverains, des opinions publiques occidentales et des actionnaires, mais aussi les mauvaises expériences du passé, encouragent les grandes entreprises sur le chemin de la Responsabilité sociale et environnementale (RSE). Ainsi, suprême paradoxe, on prétend même placer l'exploitation des ressources non renouvelables au service du développement durable, comme l'a montré l'emblématique projet pétrolier tchadien (voir Donner, 2003; Magrin, 2003, 2011). Le texte de Jessica Oder sur les mutations de la filière industrielle de l'or en Guyane française montre les efforts contrariés pour utiliser cette activité extractive rédimée au service des objectifs du développement durable à l'échelle d'un territoire.

Quelle est la portée de ces changements ? Dans quelle mesure ces nouvelles rhétoriques s'accompagnent-elles de réelles modifications des pratiques des entreprises? Et, quoi qu'il en soit, quelle est l'influence géographique actuelle des mines sur leur environnement d'accueil? Les changements technologiques font que les activités extractives n'ont plus les effets d'entraînement économique de naguère: la mécanisation des sites restreint l'emploi et les migrations (Deshaies, 2007), les codes pétroliers et miniers néolibéraux promus par la Banque mondiale, surtout en Afrique ${ }^{5}$, limitent les prélèvements et possibilités de contrôle des États (Campbell, 2009). Les mines n'ont plus le pouvoir urbanisant, socialement épais et donc structurant qu'elles eurent par le passé, par exemple dans la ceinture de cuivre de l'Afrique australe (Ferguson, 1999). L'implantation dans des milieux potentiellement hostiles incline à l'enclave plus qu'à la greffe (Magrin, van Vliet, 2005 ; Donner, 2009). Les implants extractifs procèdent de la production d'espaces extraterritoriaux qui contribuent à la fragmentation des territoires nationaux tout en étant à la base du fonctionnement de l'espace mondialisé contemporain (Antheaume, Giraut, 2005 ; Ferguson, 2006). Mais le potentiel des archipels, notamment extractifs, ne réside-t-il pas aussi dans leur capacité à s'appuyer sur le territoire, local et national, et à le structurer en retour (Arrault, 2005) ? Les lieux d'extraction font désormais l'objet d'une surveillance mondiale. Mais, dans des pays en développement, l'injonction de la société-monde de protéger et de mettre en réserve les espaces à haute valeur écologique est aujourd'hui difficilement acceptée quand ces espaces recèlent des ressources naturelles. La proposition de l'Équateur de négocier la non exploitation des hydrocarbures en Amazonie, dans le parc Yasuni, en échange d'une indemnisation mondiale, n'est pas sans intérêt. Elle pourrait d'ailleurs donner des idées à d'autres pays sommés d'arbitrer la cohabitation entre activités pétrolières et hot spot de la biodiversité, comme la Mauritanie tentée par l'exploration pétrolière sur les périphéries immédiates du Banc d'Arguin (Magrin et al., 2011). L'éthique du développement durable n'est-elle finalement destinée qu'à être instrumentalisée au service de la protection des enclaves extractives, comme l'envisage ici Nicolas Donner? Ou bien, au contraire et à l'échelle des territoires nationaux, le développement extractif dans un nouveau contexte mieux régulé, ou en tout cas ouvert au vent du débat, permettrait-il d'ouvrir des espaces de bifurcation pour les systèmes sociopolitiques rentiers hors de l'ornière de la "malédiction des ressources " (van Vliet, Magrin, 2009), à la manière de ce qui est suggéré par le Nostromo de Conrad (Magrin, 2011)?

11 Un autre changement majeur tient aux protagonistes du jeu extractif. À l'émergence d'une opinion publique nationale sur ces questions et des organisations de la société civile s'ajoute l'arrivée dans le jeu international extractif de petites entreprises (indépendantes du pétrole, junior du secteur minier) et d'entreprises des grands pays émergents. Ces deux catégories très différentes sont supposées largement 
imperméables à la RSE, car elles n'ont pas une image mondiale à défendre devant des consommateurs ou des actionnaires, comme le montre le texte de Matthieu Thune à propos de l'exploitation d'une mine d'or au Burkina Faso par une junior anglaise. L'essor des premières a été favorisé par la financiarisation qui caractérise la mondialisation contemporaine (Megret, 2011). Elles sont surtout spécialisées dans l'amont (prospection), l'exploitation de petits gisements, ou de gisements arrivés en fin de cycle, dont les coûts et conditions d'exploitation découragent les plus grandes firmes (van Vliet, 1998; Magrin, van Vliet, 2005). Les secondes ont d'abord ajouté à ce type de présence des investissements dans des environnements géopolitiques risqués ou interdits aux entreprises occidentales, comme les compagnies pétrolières chinoises au Soudan sous embargo. Puis, leur montée en puissance technologique et financière leur a permis de rivaliser avec leurs concurrents sur presque tous les types de gisement ${ }^{6}$. Ainsi, l'essor de champions nationaux chinois, indiens ou brésiliens développant des stratégies d'appropriation des réserves de matières premières jugées stratégiques pour nourrir leurs industries en pleine croissance participe de la grande mutation du monde marquée par le déclin relatif des anciennes puissances (États-Unis, Europe) et le déplacement du centre de gravité éco-démographique mondial vers l'Asie.

Ces nouveaux acteurs induisent de nouvelles relations et de nouveaux rapports de force, tant avec les pays hôtes qu'avec les entreprises occidentales. Il serait réducteur de ne voir dans ce nouveau contexte qu'une intensification de la concurrence entre entreprises: les complémentarités entre opérateurs sont nécessaires du fait de la diversité des gisements en termes de taille, de conditions d'opération (accessibilité, difficultés géopolitiques, réglementation), et de la temporalité de l'exploitation (prospection, début ou fin de cycle). Il s'agit d'approvisionner un même système. En outre, la différence fondamentale entre entreprises du Nord et des pays émergents en matière de RSE est couramment soulignée : l'extraction éthique fournirait un avantage comparatif aux premières face au danger que représentent pour elles les secondes (Soares de Oliveira, 2008). Inversement, le fait que les entreprises du Sud soient moins suspectes d'impérialisme économique leur donne un avantage pour la négociation des contrats avec des gouvernements de gauche soucieux de sortir de leur face à face inégal historique avec les grandes puissances occidentales. En témoignent par exemple la concession accordée à l'entreprise indienne Jindal Steel and Power Limited en Bolivie pour l'exploitation du fer du Mutun, ou les facilités dont bénéficient dans ces pays les investissements de Petrobras (Brésil) ou de la Petróleos de Venezuela S.A. (PDVSA) dans le secteur des hydrocarbures. Il serait pourtant réducteur d'insister sur la contribution de ces nouveaux venus à la pérennité de la "malédiction des ressources", sous prétexte que leurs standards seraient moins élevés que ceux des grandes entreprises occidentales, et que leur irruption serait favorable à une sorte de dumping social et environnemental. Les rares études disponibles sur le sujet, comme celle portant sur la régulation environnementale de la China National Petroleum Corporation (CNPC) au Tchad, suggèrent des réalités plus complexes, et l'existence de convergences significatives dans les pratiques des entreprises de toutes origines, à des rythmes et selon des modalités variables (van Vliet, Magrin (dir.) 2011). L'étude ici présentée par Romain Dittgen sur le projet chinois d'exploitation du fer de Bélinga, au Gabon, montre, de même, que la stratégie de la Chine s'accommode d'ajustements fréquents, et se révèle moins soumise à la seule logique politique et plus sensible aux signaux du marché qu'on pouvait initialement le supposer. 


\section{Cinq voyages dans l'extraction contemporaine}

13 Les cinq textes publiés dans ce dossier n'épuisent évidemment pas la complexité de la thématique ainsi sommairement présentée. Ils présentent néanmoins une variété d'objets, d'échelles et d'angles d'analyse. Quatre ressources naturelles sont ici considérées : le pétrole, le fer, l'or, les gemmes, par ordre décroissant d'importance pour le fonctionnement du système économique mondial. Les deux premières en fondent le fonctionnement - le pétrole surtout. Les deux autres relèvent d'autres logiques. L'or, en dépit de son inutilité industrielle et de sa relative abondance, a su conserver sa valeur refuge, malgré (voire contre) tous les changements de la mondialisation contemporaine. Le haut niveau de ses prix fait exploser son exploitation sur tous les continents. La géographie mondiale des gemmes est moins connue, en dehors de celle du diamant; les facteurs qui l'influencent distinguent ces pierres précieuses des autres ressources extractives. Les articles proposés ici empruntent aussi des échelles variées : celui de Matthieu Thune étudie la manière dont une mine s'insère dans son environnement local ; ceux de Jessica Oder et Romain Dittgen appréhendent les enjeux d'une filière (or en Guyane) ou d'un projet géant (fer de Bélinga au Gabon) à l'échelle de territoires de taille comparable. Enfin, les textes de Nicolas Donner et de Rémy Canavesio traitent, à l'échelle mondiale, de processus concernant un type de ressource (le pétrole, les gemmes), en mettant l'accent par les exemples retenus respectivement sur le Golfe de Guinée et sur l'Asie méridionale et l'Afrique orientale. Enfin, ils adoptent des perspectives différentes: l'impact d'un projet minier sur son milieu d'accueil (or au Burkina, voire fer au Gabon); les logiques qui permettent la sécurisation des enclaves pétrolières; les facteurs de recomposition d'une filière dans un territoire (or en Guyane) ou à l'échelle mondiale (géographie des gemmes).

Le texte de Matthieu Thune, "L'industrialisation de l'exploitation de l'or à Kalsaka, Burkina Faso: une chance pour une population rurale pauvre?", se situe dans le contexte de l'explosion de l'exploitation industrielle de l'or en Afrique de l'Ouest au cours des années 2000 (Campbell, 2009; Mbodj, 2011), dont le Burkina a constitué un des derniers eldorados. Dans un espace pauvre du sud du plateau Mossi, depuis longtemps à la limite du surpeuplement, la mine fournit-elle une opportunité pour les habitants, ou un facteur de perturbation supplémentaire? La mine s'installe sur une colline où cohabitaient assez bien agriculture et orpaillage, le second offrant une forme de diversification utile à l'économie rurale. Le texte montre comment, à Kalsaka comme souvent ailleurs en Afrique de l'Ouest, les formes de la compensation extractive sont fondamentalement différentes pour l'agriculture et l'extraction artisanale. Les droits d'usage de la première sont reconnus et indemnisés; les bénéficiaires connaissent par la suite des fortunes diverses, souvent inscrites dans les trajectoires individuelles antérieures. Les préjudices subis par l'orpaillage traditionnel ne sont pas pris en compte, malgré l'ancienneté de l'activité et l'organisation coutumière qui la régule. La différence de traitement tiendrait-elle moins à la différence entre sol et sous-sol (les deux étant, en droit, également propriétés de l'État, qui concède des droits d'usage aux habitants) qu'au rapport de ces pratiques à l'espace, plus sédentaire pour l'agriculture, mobile pour l'orpaillage ? Sur la colline de Kalsaka, l'industrie minière a donc évincé l'orpaillage filonien. Mais celui-ci était près d'arriver à une impasse, car les techniques disponibles ne permettaient plus de rechercher l'or à plus grande profondeur. Par ailleurs, l'impact économique de la mine sur son milieu d'accueil est faible : l'emploi, 
comme toujours, est limité, et presque hors de porté des riverains. Les relations communautaires de l'entreprise tiennent à quelques bonnes paroles, un peu de paternalisme et des réalisations très modestes. Les faibles salaires miniers ne suffisent pas à compenser localement le manque à gagner lié au départ des orpailleurs. Au final, la mine d'or de Kalsaka semble n'avoir constitué ni une opportunité ni une catastrophe pour les riverains. L'étude débouche sur le besoin de comparaison avec d'autres sites : la faiblesse des retombées locales est-elle due à la stratégie d'un opérateur junior (une entreprise anglaise, Cluff Gold) particulièrement peu intéressé par les pratiques de la RSE? Ou bien est-elle inhérente à l'enclavement nécessaire aux activités extractives contemporaines?

La contribution de Jessica Oder, intitulée "Vers la structuration d'une filière aurifère "durable»? Le cas de la Guyane française », traite de ressources inscrites dans la continuité géologique du socle de l'Afrique de l'Ouest, celui de l'ancien Gondwana. Cette apparente symétrie et les comparaisons qu'elle suggère ne contribuent pas peu à l'intérêt de ce texte, même si les processus étudiés s'inscrivent dans des contextes politiques radicalement différents : au lieu de l'État africain aux faibles capacités de régulation, la France ultramarine. Quand, en Afrique, la politique d'attraction des investissements miniers est justifiée avant tout par les ressources qu'elle va procurer aux États (l'emploi n'étant qu'une revendication locale des riverains des sites... source permanente d'insatisfaction), la promotion de l'exploitation industrielle moderne de l'or en Guyane est présentée comme une politique étatique, relayée par les collectivités locales, visant à répondre au besoin de diversification de l'économie et de création d'emplois face à une croissance démographique jugée très forte (à l'aune des critères hexagonaux). Les acteurs principaux de la filière aurifère moderne guyanaise sont de petites ou très petites entreprises, qui peinent à satisfaire les exigences de la puissance publique, que le Grenelle de l'environnement (2007) semble avoir soudainement convertie à la religion du développement durable. Là réside le paradoxe aurifère guyanais interrogé par J. Oder : à la différence de l'Afrique, la hausse sans précédent des prix de l'or s'accompagne d'une crise de la filière moderne, en difficulté notamment face à l'orpaillage clandestin. Est-ce parce que l'État serait trop fort, étouffant l'initiative privée à coup de mesures de régulation hors de portée de petites entreprises fragiles? Ou parce qu'il serait finalement trop faible pour accompagner les acteurs de la filière et maîtriser un vaste territoire forestier peu peuplé en proie aux infiltrations du puissant système des garimperos brésiliens? Le texte ouvre une autre perspective intéressante. Le développement de l'industrie de l'or en Guyane est mis au service de revendications politiques autonomistes: le conseil régional promeut les activités extractives dans une perspective d'empowerment et de valorisation de ressources longtemps monopolisées par le colonisateur, d'une manière qui n'est pas sans rappeler les dynamiques en cours dans la Province Nord de Nouvelle Calédonie avec le boom du nickel. Le développement par la mine doit-il alors être interprété comme un vecteur d'émancipation politique, ou au contraire comme l'adhésion à une vision métropolitaine du développement (par la modernité industrielle et les valeurs capitalistes et individualistes qui l'accompagnent) opposée à des valeurs alternatives qui avaient pu faire la force de contestations politiques autochtones?

Romain Dittgen interroge quant à lui la capacité de transformation associée au projet chinois d'exploitation du fer de Bélinga, au Gabon, dont le contrat a été signé en 2006, mais dont la mise en œuvre n'a pas encore commencé. Ce projet minier est mis en perspective à la fois par rapport à l'histoire des cycles miniers du Gabon (Pourtier, 
1989), au contexte africain des années 2000, marqué par des investissements massifs de la Chine dans les ressources extractives (Alden, Large, Soares de Oliveira, 2008), et à celle des relations sino-gabonaises. Autour de Bélinga, le besoin de diversification diplomatique et économique du Gabon rencontre l'appétit de matières premières de l'atelier du monde. L'asymétrie des acteurs en présence au moment de la discussion initiale du projet n'est pas sans évoquer celle de n'importe quelle multinationale occidentale face à des États africains désireux d'élargir leurs rentes. D'emblée, le projet revêt une dimension très politique, au sens où il est rendu possible par des discussions menées au plus haut niveau entre les États chinois et gabonais. L'entreprise chinoise est choisie face à une concurrente brésilienne dans des conditions opaques. Le projet s'insère dans le cadre d'une stratégie globale de la Chine en Afrique, qualifiée de "gagnant gagnant ", manifestée par de l'aide en nature (infrastructures) en échange du contrôle de matières premières. L'exploitation du fer de Bélinga suppose un investissement de 2,2 milliards de dollars et 4 ans de travaux de construction. Ces derniers visent à désenclaver des gisements situés dans une région quasiment déserte par un chemin de fer, à construire des barrages pour fournir de l'énergie, un port minéralier pour traiter 20 millions de tonnes annuelles... L'auteur analyse les effets d'entraînement potentiels sur le territoire national et la région de production. L'incertitude qui entoure la réalisation du projet est finalement étudiée : la crise de 2008 et la baisse du prix du fer y ont leur part; l'incertitude politique au Gabon a la sienne, à la suite de la mort d'Omar Bongo, qui avait personnellement contribué au rapprochement avec les Chinois. Elle fut propice à l'expression de critiques de la part d'ONG et de médias nationaux concernant les termes du contrat jugés défavorables à l'État, aux populations locales et à l'environnement. Le retard du projet conduit surtout à questionner la soi-disant spécificité des acteurs chinois, pour lesquels la rentabilité de court terme serait secondaire par rapport aux visées stratégiques de long terme, ce qui expliquerait la prépondérance des relations politiques sur les calculs financiers lors de la négociation des projets. Le projet de Bélinga évoque aussi celui du fer de la Falémé, au Sénégal, où le géant sidérurgique indien Mittal obtint un permis en échange de promesses d'investissements lourds en infrastructures et d'emplois locaux, avant de se dédire à la suite de la crise de 2008. En Bolivie, le choix de l'entreprise indienne Jindal Steel pour exploiter le fer du Mutun (40 milliards de tonnes) donne lieu aujourd'hui à des débats publics analogues sur la capacité de l'entreprise à organiser une telle exploitation et sur les faveurs dont elle aurait bénéficié de la part de l'État. Quoi qu'il en soit, la rentabilité inférieure de l'exploitation minière par rapport à celle du pétrole explique les stratégies mondiales difficiles à déchiffrer des entreprises géantes, aujourd'hui souvent chinoises, indiennes ou brésiliennes. Celles-ci concilient calculs à très long terme et prise en compte des cycles courts de la conjoncture, pour tantôt se contenter de contrôler des gisements de réserve, tantôt franchir le pas qui mène à leur exploitation. De telles stratégies n'en soulignent que mieux la position de faiblesse des États hôtes dans la négociation des conditions de l'exploitation.

17 La contribution de Nicolas Donner file la métaphore médicale pour décrire les modalités d'insertion des installations d'extraction pétrolière dans leur milieu d'accueil. Partant du constat du paradoxe pétrolier selon lequel la mobilité du monde repose sur une matière puissamment inerte, ces « Notes sur la dimension immunitaire des enclaves pétrolières " décortiquent le dispositif permettant l'immunisation du système pétrolier vis-à-vis des dangers potentiels de son milieu hôte, condition de la mise en circulation de la ressource. Il mobilise pour ce faire divers exemples d'enclaves 
dans le monde, mettant l'accent sur celles installées dans les espaces les plus hostiles, les zones vierges et les milieux à basse gouvernabilité, notamment en Afrique. Deux modalités principales de cette immunisation sont présentées. Certaines enclaves pétrolières sont construites par un affranchissement le plus complet possible des conditions environnantes. Le cœur de celles situées en milieu vide en constituant un archétype, à mi-chemin entre les formes urbaines les plus modernes (produisant un milieu presque entièrement artificialisé) et les capsules spatiales (construisant un espace de vie étanche). Les enclaves pétrolières s'assimilent ainsi, de façon plus ou moins parfaite, au modèle de l'île absolue décrit par Sloterdijk (2005). Elles illustrent aussi une forme paradoxale d'être au monde désormais classique caractérisée à la fois par l'isolation (vis-à-vis de l'alentour immédiat) et la connexion (au reste du monde, et surtout aux pôles de l'archipel). Le spectre des techniques d'immunisation est large, et recouvre jusqu'aux vêtements du "pétronaute »: les manches longues anti-paludisme portées par le personnel du projet Exxon au Tchad par exemple. Dans les espaces habités, surtout lorsqu'ils relèvent de milieux géopolitiquement sensibles appartenant à des États "fragiles", selon la terminologie des institutions de Bretton Woods, le processus d'immunisation repose sur une double fonction défensive et interventionniste. La première relève des gangues successives qui enveloppent les installations (protections, murs, zones d'accès sélectif) pour les protéger. L'épaisseur de ces clôtures varie selon les situations, mais elles ne sont jamais totalement hermétiques, pour permettre la respiration du corps extractif, et une certaine plasticité de relations avec l'extérieur. La seconde fonction concerne la maîtrise de relations actives, avec et non plus sans le milieu d'accueil. C'est là que l'arsenal lexical, idéologique et méthodologique de la RSE et du développement durable peut se déployer avec succès. D'une manière plus large, l'article s'interroge sur les fonctions de l'idéologie du développement durable autour des systèmes extractifs. Ce paradigme dominant ne serait pas seulement une réponse plus ou moins aboutie aux indignations suscitées par les impacts les plus négatifs des activités extractives dans les années 1990. Il participerait plus fondamentalement des stratégies d'immunisation mises en œuvre par les entreprises et les bailleurs de fonds qui accompagnent le système extractif pour la satisfaction des besoins des pays dominants. Ainsi, le paradigme du développement durable couplé aux stratégies de stabilisation et de légitimation locale rencontre aussi bien les stratégies de sécurisation des approvisionnements énergétiques que la sensibilité de l'opinion publique occidentale. Les vertus de l'éthique sont instrumentalisées au service de la «social licence to operate » et de la sécurisation des zones de production - ce qui n'empêche pas, en pratique, de sérieux tâtonnements dans la mise en œuvre. Le projet Tchad Cameroun soutenu par la Banque mondiale constitue un bon exemple de cette logique et de ses limites. Le texte est tout entier construit autour du point de vue du système pétrolier et de sa terminaison, le greffon extractif. Inverser la perspective, en considérant les intrusions pétrolières depuis le milieu d'accueil, et la manière dont elles servent de support, à différentes échelles, à des constructions imaginaires et à des stratégies d'acteurs, pourrait en représenter un prolongement intéressant. Enfin, on peut se demander dans quelle mesure cette lecture des procédures d'immunisation des enclaves pétrolières serait applicable aux autres formes d'extraction : pour petites qu'elles soient, les mines d'or ou de diamant peuvent leur ressembler. Mais les très grands projets miniers impliquant des infrastructures lourdes reposant sur le territoire et de nombreux emplois dessinent sans doute un modèle géographique légèrement différent. 
18 Enfin, le texte de Rémy Canavesio, "Croissance économique des pays émergents et géographie mondiale des pierres précieuses", concerne des ressources largement méconnues, aux propriétés pourtant originales. À l'aune du système mondial des hydrocarbures, c'est une économie de pacotille : les montants financiers concernés sont incomparables, et la finalité peut être tenue pour futile, voire simplement symbolique. Mais la géographie des gemmes est complexe, et structure des filières mondiales aux effets géographiques localement importants. Les lieux d'extraction, les filières et marchés varient selon les pierres. Si l'article met en perspective les dynamiques de l'ensemble des systèmes gemmifères, il ne met pas l'accent sur le diamant, le mieux connu, mais sur les saphirs et rubis, qui le sont beaucoup moins. Il s'agit d'abord de comprendre les facteurs qui influencent leur géographie : ils ne sont pas géopolitiques ou stratégiques, comme pour le pétrole. À la différence de l'or, les filières des gemmes ne sont guère sensibles à la libéralisation des codes miniers, car elles demeurent largement artisanales et informelles. En revanche, les pôles de consommation s'expliquent en partie par des héritages historiques et culturels, et leurs évolutions tiennent beaucoup aux grandes dynamiques macro-économiques. La demande mondiale de gemme ressemble à celle des automobiles: elle est tirée par l'enrichissement des pays émergents, alors que les marchés occidentaux sont «matures» depuis longtemps. Le diamant, largement déterritorialisé dans sa production comme dans sa consommation, bénéficie de chaque enrichissement rapide d'une population importante (voir Brunet, 2004). Or, l'enrichissement des pays émergents géants ne se traduit pas dans des termes homogènes : si les Chinois aiment le jade, leur soif de consommation (occidentale?!) devrait les porter bientôt vers le diamant, à la façon nippone. En Inde en revanche, des résistances culturelles vivaces à l'occidentalisation se traduisent notamment par une inclination pérenne pour les rubis et saphirs. En outre, l'enrichissement transforme aussi la géographie de l'offre. L'extraction des gemmes étant largement artisanale, la forte valeur des pierres suffit à rendre l'activité rentable pour des populations pauvres, même avec des gisements à faible concentration. L'extraction artisanale contribue ainsi à des systèmes régionaux d'activité qui contrastent fortement avec les enclaves extractives industrielles. De la ceinture des diamants de l'Afrique de l'Ouest à celle de l'Afrique centrale (RCA ; Kassaï Nord-Est angolais), des émeraudes et améthystes de Colombie et du Minas Gerais (Brésil), des saphirs et rubis du sud du Sri Lanka, de la frontière thaïlandocambodgienne à la région de Mogok en Birmanie, la géographie de l'extraction artisanale est celle de régions pauvres, souvent enclavées ou isolées, parfois en conflit ou post-conflit. Les facteurs qui influencent les dynamiques des espaces gemmifères ne se limitent pas à la confrontation entre une demande solvable et une offre conditionnée par les faveurs de la géologie. Il faut que les concentrations connues soient trop faibles pour justifier la mécanisation, et que le niveau de pauvreté ou des crises demeure à un niveau suffisamment élevé pour maintenir l'attractivité d'une activité pénible, dangereuse et à la rentabilité aléatoire. La forte demande asiatique en saphirs et rubis ne suffira pas à empêcher la baisse de la production thailandaise, victime de l'enrichissement relatif du pays. L'Afrique orientale (Tanzanie, Kenya, Madagascar, voire Somalie), qui compte de nombreux petits gisements dispersés, et des conditions politico-économiques médiocres, pourraient être sollicitée pour répondre à la demande à venir, et connaître en conséquence le plus grand nombre de nouvelles ruées.

$19 * * *$ 
En dépit de sa richesse, ce dossier laisse de côté nombre de thèmes importants: certaines aires géographiques en sont absentes, comme le Moyen-Orient, la Russie, les pays du Nord, de même que, en dehors de la Chine, les grands pays émergents ; la déclinaison géographique des nouveaux nationalismes énergétiques n'y parait guère, de même que des analyses sur les risques environnementaux ou les paysages extractifs. La pétropolitique et les "guerres de ressources » offrent également des champs larges peu abordés ici. Ce numéro d'Echogéo aura posé quelques balises d'une thématique à laquelle la promesse de la transition énergétique conférera une importance encore plus centrale dans les années à venir. Car si nul ne peut encore connaître le rythme et les contours de cette transition, on peut deviner l'ampleur des implications d'une telle redistribution des cartes de l'énergie et donc du pouvoir.

\section{BIBLIOGRAPHIE}

Alden C., Large D., Soares de Oliveira R. (eds.), 2008. China Returns to Africa. A rising Power and a Continent Embrace. $382 \mathrm{p}$.

Antheaume B., Giraut F. (dir.), 2005. Le territoire est mort, vive les territoires! Une (re)fabrication au nom du développement. Paris, IRD Editions, 384 p.

Arrault J.B., 2005. Du toponyme au concept ? Usages et significations du terme archipel en géographie et dans les sciences sociales. L'Espace géographique, 2005-4, p. 315-328.

Bauman Z., 2006. La vie liquide, trad. C. Rosson. Rodez, Le Rouergue/Chambon ( 1 ère édition, Liquid modernity (2000)).

Bayart J.F., 1989. L'État en Afrique. La politique du ventre. Paris, Librairie Erthème Fayard, $2^{\mathrm{ème}}$ édition, 2006, 439 p.

Brunet R., 2004. Le diamant. Un monde en révolution. Paris, Belin, 416 p.

Campbell B. (ed.), 2009. Mining in Africa. Regulation and Development. London, New York, PlutoPress, International Development Research Center, The Nordic Africa Institute, 276 p.

Donner N., 2003. «Chad Development Project ». Approche d'un projet pétrolier à l'heure du développement durable. Le projet Exxon Mobil au Tchad. Mémoire de maîtrise de géographie, université Joseph Fourier, Grenoble I, 166 p.

Donner N., 2009. The Myth of the Oil Curse: Exploitation and Diversion in Equatorial Guinea. AfroHispanic Review, vol. 28, n² 2, p. 21-42.

Ferguson J., 1999. Expectations of modernity. Myths and meanings of urban life on the Zambian Copperbelt. Berkeley, Los Angeles, London, University of California Press, 326 p.

Ferguson J., 2006. Global shadows. Africa in the neoliberal World Order. Durham and London, Duke University Press, $257 \mathrm{p}$.

Gabriel-Oyhamburu K., 2010. Le retour d'une géopolitique des ressources ? L'Espace Politique [En ligne], 12 | 2010-3, mis en ligne le 11 février 2011, Consulté le 08 septembre 2011. URL : http:// espacepolitique.revues.org/index1796.html 
Gottmann J., 1952. La politique des États et leur géographie. CTHS, 261 p.

Hönke J., 2010. New political topographies. Mining companies and indirect discharge in Southern Katanga (DRC). Politique africaine, ${ }^{\circ} 120$, décembre 2010, p. 105-128.

Magrin G., 2003. Les enjeux d'un enrichissement pétrolier en Afrique centrale. Le cas du Tchad. Paris, Grafigéo $n^{\circ} 22$, coll. Mémoires et documents de l'UMR PRODIG, 93 p.

Magrin G, van Vliet G., 2005. Greffe pétrolière et dynamiques territoriales : l'exemple de l'on shore tchadien. Afrique contemporaine, $\mathrm{n}^{\circ} 216$, p. 87-105.

Magrin G., van Vliet G., van Dessel B., Chabason L., 2011. La Mauritanie et la mer : et si le pétrole aidait à mieux gérer l'insécurité écologique? Natures, Sciences et Sociétés, 3/2011 (sous presse).

Magrin, 2011. Des rentes aux territoires. Contribution pour une géographie du développement en Afrique. Dossier d'Habilitation à diriger des recherches, université Paris 1 Panthéon Sorbonne, volume 3, essai, $447 \mathrm{p}$.

Martinez L., 2010. Violence de la rente pétrolière : Algérie - Irak - Libye. Paris, Presses de la fondation nationale des sciences politiques, $229 \mathrm{p}$.

Mbodj F.B., 2011. Boom aurifere à l'est du Sénégal, à l'ouest du Mali et au nord-est de la Guinée : mutations socio-économiques et spatiales d'anciennes marges économiques et géographiques. Thèse de doctorat de géographie, université Paris 1 - Panthéon Sorbonne, mai 2011.

Megret Q., 2011. De l'inscription en bourse à l'exploration en brousse. La double vie d'une multinationale minière junior. Carnets de géographes [En ligne], Numéro 2, mars 2011, rubrique Carnets de terrain, consulté le 5 septembre 2011 http://www.carnetsdegeographes.org/ carnets_terrain/terrain_02_04_Megret.php

Perrier-Bruslé L., 2008. Le gaz bolivien. La Bolivie face à son avenir. Outre-Terre, Revue française de géopolitique, Eres, $\mathrm{n}^{\circ}$ 18, 2007/1, p. 235 - 251 http://www.cairn.info/revue-outreterre-2007-1-p-235.htm

Pourtier R., 1989. Le Gabon, tome 2, État et développement. Paris, L'Harmattan, 344 p.

Raffestin C., 1980. Pour une géographie du pouvoir. Paris, Librairies techniques, 249 p.

Rosser A., 2006. The Political Economy of the Resource Curse: A Literature Survey. IDS Working paper $\mathrm{n}^{\circ} 268$, Centre for the Future State, $34 \mathrm{p}$.

Sloterdijk P., 2005. Écumes (Sphères III). Paris, Hachette littératures.

Soares de Oliveira R., 2007. Oil and Politics in the Gulf of Guinea. London, Hurst \& Company, 379 p.

Soares de Oliveira R., 2008. Making sense of Chinese Oil Investment in Africa, Alden C., Large D., Soares de Oliveira R. (eds.), China Returns to Africa. A Rising Power and a Continent Embrace, Londres, Hurst \& Company, p. 83-109.

van Vliet G., Magrin G. (dir.), 2012. La Chine sur la piste du pétrole africain : la CNPC et les enjeux environnementaux au Tchad. AFD, collection Focales, Quae (à paraître en 2012).

van Vliet, G., 1998. Activités minières, barrages, et problématiques de négociation : hypothèses pour une réflexion. 1998, présentation ppt.

van Vliet G., Magrin G., 2009. Public steering in the hydrofuel sector. Conditions for trajectory bifurcation in Chad and Mauritania. $21^{\text {st }}$ world Congress of Political Science, Santiago du Chili, 12-16 juillet 2009. 


\section{NOTES}

1. EchoGéo n8, mars mai 2009 http://echogeo.revues.org/10263

2. Autour d'historiens du CNAM et de l'EHESS (France), un groupe de chercheurs s'est constitué depuis 2009 pour travailler sur les « débordements industriels » dans la cité et les conflits qu'ils suscitent. La notion de « débordements industriels ", qui reste à préciser, embrasse les accidents, pollutions, dangers, risques associés à l'activité industrielle. Les activités extractives y ont une part significative. http://www.debordementindustriel.fr/modeles/presentation.htm

3. Voir la Libre tribune d'Alain Juppé dans le dossier "Afrique, nouvelle terre de conquête", Diplomatie n52, septembre 2011 : « l'Afrique doit être notre « nouvelle frontière » " (p. 10).

4. Cette notion traduit l'idée que, dans les États «fragiles", la rente pétrolière fournirait à de minces élites libérées de toute responsabilité politique et sociale envers leurs concitoyens les moyens de se maintenir indéfiniment au pouvoir, en finançant les moyens de la répression et en garantissant un soutien international inconditionnel et multiforme.

5. En Amérique latine, à partir du milieu des années 2000, la nationalisation des hydrocarbures en Bolivie et des renégociations des contrats en Équateur et au Venezuela dessinent plutôt un contexte post-néolibéral.

6. Dans le domaine pétrolier, l'offshore ultra-profond demeure l'apanage des majors occidentales. Si la Brésilienne Petrobras y a fait récemment une percée remarquée, les entreprises chinoises ou indiennes y demeurent encore en retrait.

\section{INDEX}

Thèmes : Sur le Champ - Sur le Terrain 